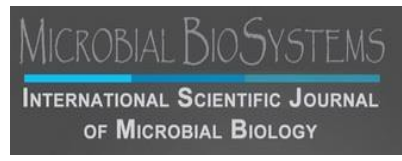

\title{
Lichens in folklore: medicine and dyes
}

\author{
Abdel-Fattah AF \\ Botany Department, Faculty of Science, Suez Canal University, Ismailia 41522, Egypt.
}

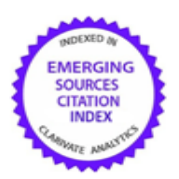

Published by Arab Society for Fungal Conservation

\section{Lichens in medicine}

Lichens were mentioned in folklore of different cultures around the world for its ability to treat several diseases; thus, it has been used in folk medicine.

Lichens were believed to be useful in the treatment of wounds, skin diseases, gastrointestinal and respiratory problems. It has been reported that lichens characterized by high carbohydrate storage, contain several secondary bioactive metabolites that act as antibiotics. Lichens were used topically as wound disinfectants, anti-bleeding, other skin problems such as soreness and mouth infections.

According to Müller (2002), lichen metabolites possess a wide range of biologically active compounds with antibiotic, antifungal, antiviral, anti-inflammatory, analgesic, antipyretic, antitumor, immunomodulation and cytotoxic potentials. Despite of these different activities of lichen metabolites, their therapeutic potentials have not fully investigated yet and remain pharmaceutically untapped. We can conclude the importance of lichens usage from the word leikhēn, "what eats around itself", which derived from ancient Greek custom of using a cryptogam to treat skin disorders. Lichens are often drunk as a decoction for treating lung or digestive-related ailments. Many other lichens uses related to obstetrics or the treatment of gynecological problems. This may be due to the widespread utilization of lichens to cure infections that transmitted sexually and urinary system disorders.

In many different cultures, there are other uses of lichens for the treatment of eye afflictions, the treatment of feet problems and the use of smoking mixtures.

Usnea is the most commonly used genus of lichen, however it is often used synonymously with other arboreal hair lichen. Usnea is a long, pendulous and hair like species used for scalp disorders and hair reinforcement.

\section{Lichens and dyes}

Some lichens contain acids which may produce a number of dye colours when treated. There are basically two major types of dyes that are derived from lichens. Orchil is a red dye, recognized as acid-alkalin indicator litmus, which is produced from Roccella, Ochrolechia, and others. Orcien which is the colouring matter of orchil that derived from deposition of orcinol, particularly erythrin, lecanoric and gyrophoric acid. According to Zidan (1987), this dye produced from sea lichens found in Mediterranean Sea rocks. It is used as a basis for the highly valued purple tyrian that secreted by shellfish.

Crottle is a rich colour of yellow-brown to red-brown used for wool dying, leaving it soft and fragrant. In contrast to cudbear, crottle dyes are very light fast and it is shocking that they have never been processed on a large commercial scale. This dye derived from B-orcinol depsidones, especially salazinic acid. Noteworthy, that this dye was much darker years ago.

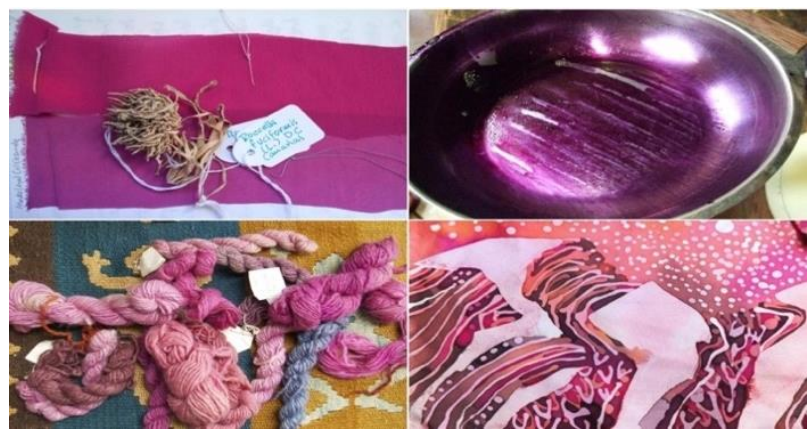

Orchil or Archil purple dye made from lichens 


\section{Further readings}

Abdel-Kareem O (2012) History of dyes used in different historical periods of Egypt. Res J Text Apparel 16(4): 79.

Malhotra S, Subban RAVI, Singh A (2008) Lichens-role in traditional medicine and drug discovery. Inter J Alter Med $5(2): 1-5$.

Coppins BJ, Watling R (1995) Lichenized and non-lichenized fungi: Folklore and fact. Bot J Scot 47(2): 249-261.

Crawford SD (2019) Lichens Used in Traditional Medicine. In: Ranković B. (eds) Lichen Secondary Metabolites. Springer, Cham, pp 31-97.

\section{ARTICLE INFO}

Article history

Received 1 June 2020

Received revised 17 June 2020

Accepted 27 June 2020

Available online 30 June 2020

(C) Abdel-Fattah 2020

Corresponding Editor: Balbool BA, Abdel-Azeem MA 